\title{
Les écrivaines de récits de voyage du Québec: esprit féminin et lieux du savoir chez Jacqueline Darveau (1938) et Marie-Ève Martel (2011)
}

\author{
Mariève Maréchal \\ Université d'Ottawa
}

L'intérêt principal d'une recherche sur les récits de voyage des femmes, hormis le fait que le champ est très peu couvert, est bien exprimé par Bénédicte Monicat: "[l]e récit de voyage constitue [...] un apport essentiel à la littérature des femmes et à l'histoire des femmes. Il révèle l'écriture comme un domaine où la femme résiste et construit une autre histoire.» (p.129130) Le récit de voyage est donc pour elles l'occasion de transformer certaines normes sociales et discursives afin de 
s'afficher en tant que sujets dans la mémoire collective. La question se pose alors de savoir quelles seraient les modalités de résistance spécifiques aux écrivaines de voyage du Québec. Comment, pour reprendre les mots de Monicat, celles-ci construisent-elles une autre histoire? Je propose de répondre à ces questions en analysant Randonnée au pays des totems de Jacqueline Darveau, publié en 1938, relatant sa pérégrination de la ville de Québec à celle de Juneau, en Alaska, et Whisky berbère de Marie-Ève Martel, publié en 2011, racontant ses séjours au Maroc. Éloignés de soixante-treize ans, ces deux récits, en plus de témoigner de la présence, au Québec, d'une littérature de voyage au féminin, me permettra, en examinant les lieux du savoir qu'elles construisent dans leur récit, de saisir ce qui les motive à voyager et à écrire leur voyage. Dans la continuité de la pensée de Monicat, j'avancerai comme hypothèse que ces deux femmes ont élaboré leur récit dans un certain esprit de résistance, résistance qui se déploie dans mon corpus à travers la narration, la structure et l'agencement du texte. Pour ce faire, j'étudierai d'abord la voix narrative construite par ces auteures, ensuite la manière dont elle se déploie à travers la description et, enfin, je mesurerai brièvement son écho dans la forme structurelle. Ces aspects seront envisagés en même temps chez l'une et chez l'autre plutôt qu'une à la suite de l'autre dans le but de faire ressortir plus clairement les différentes pratiques d'écriture de ces femmes. Je spécifie que j'aurai une approche féministe, c'est-àdire une perspective qui prend en compte que les femmes, pour se valoriser en tant que sujets, doivent se rebeller contre différentes institutions patriarcales qui briment leur autorité discursive (Marina Yaguello, Susan Sniader Lanser, Barbara Havercroft, Patricia Smart, Lucie Joubert). Je serai aussi consciente 
que le genre sexuel influe sur notre utilisation de la langue et qu'il est ainsi «une catégorie d'étude fondamentale de l'expérience humaine et de l'organisation du savoir » (Carle, p. 11).

\section{La modalité narrative :}

\section{deux types et deux usages de la voix collective}

Selon Susan Sniader Lanser, les modes narratifs sont autorisés par des conventions sociales et littéraires qui consacrent l'autorité de la parole des hommes blancs de classe aisée et régissent, pour les femmes, leur accès à une parole publique (p. 6-7). Cette utilisation réactive donc une idéologie patriarcale sur les rapports entre les sexes. Lanser affirme toutefois que certaines écrivaines n'usent pas de façon conventionnelle des modes narratifs officiels : c'est ce que je perçois dans les textes de Darveau et de Martel. Ces femmes modifient ces modes de façon à y construire et y valoriser leur propre autorité discursive : " [they] construct narrative voices that seek to write themselves into Literature without leaving Literature the same » (p. 8). Lanser évoque plus particulièrement trois voix : la voix auctoriale, utilisant le "elle», la voix personnelle, usant du «je», et la voix collective (communal voice), qui utilise le «nous» ou plusieurs «je». Darveau et Martel construisent dans leurs écrits respectivement ces deux types de voix collective; j'examinerai comment celle-ci nous renseigne sur leur entreprise d'écriture.

À travers son récit, même si elle utilise certaines fois le « je » comme sujet de l'énonciation, Darveau investit surtout le «nous » afin de s'affirmer à travers le texte. Ce pronom, selon Anne-Marie Carle, était principalement 
employé dans les récits des religieuses ou dans ceux de quelques chroniqueuses (qui insistaient souvent, et dès les premières lignes du récit, pour dire qu'elles ne se déplaçaient pas seules) [alors] [qu']apparaît avec les voyageuses de l'entredeux-guerres un «je» qui [...] prend une place particulièrement importante au sein de la narration (p. 106).

Bien que ce ne soit pas encore vraiment le cas avec Darveau et qu'elle fasse donc figure à part, son «nous » est d'une grande polyvalence. Elle l'utilise afin de voiler sa subjectivité à travers celle de ses parents avec lesquels elle se déplace. Ainsi, elle concentre les lectrices et les lecteurs sur le voyage plutôt que sur elle en tant qu'auteure et voyageuse. Elle évite de mettre directement l'accent sur sa personne dans son récit et semble de cette manière obéir à un devoir de réserve que les discours sociaux de l'époque affirmaient être le propre des femmes. Cette utilisation souligne aussi le fait qu'elle n'est pas seule, que ses tuteurs sont avec elle, qu'elle ne s'est pas complètement affranchie, pas complètement arrachée à sa "condition" de femme par ce voyage, ce qui peut certes lui retirer une certaine crédibilité en tant que voyageuse, mais qui lui confère aussi une image de jeune femme respectueuse des traditions et des conventions. Il apparaît donc que Darveau use du «nous » afin de légitimer ses écrits. Toutefois, l'auteure affirme clairement dans l'avertissement de son récit qu'elle «désire » écrire son voyage (p. 5) en s'appuyant notamment sur les propos d'Anne Lindbergh, une célèbre écrivaine et aviatrice états-unienne, lien que j'examinerai un peu plus loin. Darveau a donc la volonté de passer outre certaines limitations qu'on impose aux femmes afin de s'exprimer. Par là, on comprend que son «nous » a un double rôle : celui de lui donner l'apparence de rester dans les normes établies en optant pour une voix impersonnelle, d'une part, et, d'autre part, celui d'assumer une autorité narrative. En 
effet, l'utilisation que Darveau fait de son «nous» n'a rien d'innocent. On constate à travers la lecture que les parents de l'auteure sont non seulement écartés comme sujets à travers ce pronom, mais aussi effacés; leur parole est tout simplement absente. Le «nous» de Darveau est indubitablement le déguisement que revêt son véritable «je». C'est une stratégie d'écriture qui lui permet de voyager et d'écrire tout en restant dans les conventions, une ambivalence que Carle évoque en termes de parole double, constituée d'un propos explicite et d'un langage implicite et qui est une caractéristique importante, selon elle, de l'écriture du récit de voyage au féminin de 1859 à 1940 (p. 10). Je note aussi que la relation à l'altérité de Darveau n'est pas très développée, et cela, du fait que la voix narrative qu'elle construit nous informe qu'elle doit déjà se battre pour légitimer sa parole en tant que femme.

Chez Martel, la narration est radicalement différente. Le fait est qu'elle voyage seule, ce qui est encore peu commun pour une femme du Québec et c'est là un point de résistance aux normes sociales. Ainsi, au contraire de Darveau, le « je » est omniprésent dans Whisky berbère. En fait, plusieurs « je », dont celui de l'auteure, se côtoient, à travers de nombreux dialogues qu'elle entretient au gré de ses rencontres. La voix narrative construite dans ce récit par Martel se manifeste donc à travers une voix collective, plus particulièrement sous sa forme séquentielle « in which individual members of a group narrate in turn » (Lanser, p. 21). Dans le récit qui m'intéresse, la parole n'est pas transmise de manière précise, mais au gré des rencontres de l'auteure. Martel accorde aux gens une autorité discursive en plaçant sa voix en retrait afin de ne pas imposer son système de valeurs et ses opinions sur eux. Cela donne l'impression que plusieurs personnes en plus de l'auteure 
racontent le récit. Martel crée donc une polyphonie narrative et valorise ainsi une subjectivité dialogique, plurielle. Il est ainsi plus facile de s'identifier à elle et aux gens qu'elle rencontre qu'avec le «nous » de Darveau. En outre, cette narration trompe les apparences et on en vient en considérer que Martel n'est jamais seule et l'auteure apparaît ainsi moins subversive. Lorsqu'elle élabore cette voix narrative, je constate qu'il est primordial pour elle d'enrichir son existence, sa subjectivité, de celle de l'Autre. Je considère alors que la formation d'une telle voix narrative constitue pour l'auteure une stratégie qui lui permet à la fois de rester dans la norme et d'afficher comment elle conçoit sa subjectivité et sa relation à autrui. Il semble ainsi que la constatation de Carle, au sujet de la parole double chez les écrivaines de voyage, vaut aussi pour un corpus plus un peu plus moderne que le sien.

\section{La description : de l'intertextualité à la théâtralisation}

Selon Véronique Magri-Mourgues, « la description [dans le récit de voyage] est légitimée d'emblée au nom de la démarche didactique que ce type de récit adopte, se voulant compterendu, véhicule d'informations» (p. 2). Chez les auteures de mon corpus, la description dénote à la fois deux types de véhicules et d'informations.

Dans le récit de Darveau, la description prend la forme d'une liste ou d'un intertexte et procure des informations de l'ordre du domaine public. Dans le premier cas, elle vise à dresser des portraits géographiques, historiques et économiques des régions et des villes qu'elle traverse. Les descriptions sont notamment élaborées à partir de documents 
statistiques dont l'auteure nous cite des données qui s'étalent souvent sur une page complète. Par exemple, Darveau écrit :

[d]'après les dernières statistiques qui m'ont été fournies par le "Bureau of foreign and domestic commerce» de Washington, l'Alaska a importé, en 1937, 8,995,461 livres de viande, 1,750,638 douzaines d'œufs, au-delà d'un million et demi de livres de beurre; des fruits et des préparations de fruits pour une valeur de $\$ 750,425.00$. La gazoline et autres produits pétrolifères au coût de $\$ 3,298,671$.00. Les habitants ont un souci de la propreté, ayant importé 1,297,916 livres de savon » (p. 78)

Les informations sont très précises, citées par un esprit méticuleux et soucieux d'exactitude. Darveau cherche à pourvoir son récit d'un discours de spécialiste. La lectrice et le lecteur sont simplement dépassés devant de telles précisions, ce qui confère une certaine autorité discursive à l'auteure.

Il en va de même dans le deuxième cas, l'intertextualité, utilisée pour construire des descriptions. En effet, les gens auxquels Darveau se réfère, des littéraires comme Anne Lindbergh, Damase Potvin, Louis Hémon, Dante Alighieri; mais aussi des scientifiques, plus particulièrement des botanistes ou des géographes comme Jules Romais, Claude Melançon, David Douglas, Edward Martin Kindle et Mrs. George Black, tiennent des discours spécialisés, donc consacrés par une institution. Anne-Marie Carle explique que l'utilisation de l'intertexte constitue «une appropriation d'un discours érudit» (p. 79) dans les récits de voyage des femmes et que

ce phénomène d'intertextualité n'est pas que substitution : il est l'occasion de cautionner une écriture peu valorisée (celle des femmes) par une écriture reconnue (celles des auteurs classiques) et de conférer à une femme une compétence en tant que voyageuse (par la qualité de sa documentation) et en tant qu'écrivaine (par ses connaissances livresque et littéraires) (p. 83). 
La parole des auteurs et des spécialistes cités a une valeur sociale qui peut servir de référents aux yeux du plus grand nombre. Darveau s'appuie donc sur ces auteurs afin d'accorder plus de crédibilité autant à son écriture qu'à son voyage, participant ainsi à construire une autre histoire que celle véhiculée par les normes et les conventions sociales de son époque portant sur les femmes, leur attitude et leur capacité.

Chez Darveau, cette autre façon d'écrire et de mettre en scène la réalité passe aussi par le choix d'intertextes particuliers. Par exemple, le premier auquel elle se réfère dans son récit provient d'une voyageuse et pilote états-unienne, Anne Lindbergh, déjà nommée, et qui a mis par écrit certains de ses voyages. Lindbergh lui sert à expliquer et à la fois à légitimer le fait qu'elle éprouve, en écrivant son voyage, une « joie personnelle » (Darveau, p. 5) comme

[c]elle des gens qui aiment à raconter leurs aventures, qui remplissent des carnets, qui content des histoires, qui écrivent des lettres; une drôle de race de gens, qui ont l'impression qu'on leur a volé la moitié d'un événement quand ils ne l'ont pas raconté (Lindbergh citée par Darveau, p. 6).

À partir de cette citation, on peut comprendre que Darveau prône la liberté des femmes, particulièrement celle de se déplacer et d'écrire, car elle inscrit son récit, et ce, avant même son début puisque Lindbergh est citée dans l'avertissement, à partir d'une lignée de femmes dont l'aviatrice et écrivaine fait figure de modèle. Une telle utilisation de l'intertexte pour décrire son voyage (ici, le désir de le mettre par écrit) constitue à mon avis un point de résistance au discours hégémonique de son époque. 
Il est à noter que, dans les descriptions de l'auteure, on remarque une relation à l'espace particulièrement significative : Darveau nous informe que c'est le voyage géographique qui importe pour elle, c'est-à-dire le déplacement, la visite de nouveaux lieux et la vue de nouveaux paysages. La circulation dans l'espace est pour elle une occasion de valoriser publiquement son existence, une sphère encore peu investie par les femmes. Darveau suggère qu'il est essentiel que sa parole ne soit plus confinée à la sphère privée et dévoile alors l'enjeu de l'accès à la parole publique pour les femmes.

Martel, soixante-treize années plus tard, opère tout autrement. Dans Whisky berbère, la description se présente sous la forme d'une mise en scène ou d'une théâtralisation du voyage. Ce trait d'écriture a été noté par Brigitte Malenfant chez certaines voyageuses; il indique « un glissement de la réalité au rêve, de l'image à l'imaginaire et fait du texte le récit du monde imaginaire de la voyageuse » (p. 78). Ainsi, la description ne vise pas ici à partager une réalité statistique ou bien à mettre de l'avant le discours de certains spécialistes. Bien au contraire, il s'agit de raconter son voyage à sa manière, à travers sa propre perception. Chez Martel, la description s'attarde sur des cas particuliers, sur ce qui est du domaine privé, voire de l'intime : elle nous informe sur ce qui se dit ou se fait autour d'une table d'un café, dans une fête privée, dans un hammam ou encore avec un amant venu la retrouver un moment au Maroc. L'auteure attire ainsi l'attention sur l'expérience humaine individuelle, celle des autres ou bien la sienne plutôt que sur son voyage, sur les villes ou sur les paysages comme c'est le cas chez Darveau. Les descriptions de Martel cherchent à éclairer des subjectivités. D'ailleurs, l'auteure repousse dans la marge, en notes de bas de page, et cela sans aucune référence, les 
aspects les plus techniques et certaines spécificités langagières qu'elle doit expliquer à un lectorat occidental qui les ignorerait. Cela montre entre autres que Martel subordonne au domaine privé ce qui a trait au domaine public.

On remarque qu'une des mises en scène les plus frappantes chez cette auteure réside dans son utilisation importante d'onomatopées afin de décrire certaines scènes de son voyage. On en trouve neuf différentes dans une seule page, reproduisant le bruit du trafic urbain et des gens ou des animaux qui y déambulent: «Put! Puuuttt!», «bzzzttt!», «Pipipipiii!» «miaowww!», «pin-pon! pin-pon! bip! bip! biiippp! », « ding! ding! », « iii! » et « ptchttt! ». Elle semble ainsi endosser le rôle d'une conteuse devant un auditoire à conquérir. Cette transcription de sons pourrait être interprétée comme une théâtralisation. Elle sert à accorder une certaine crédibilité à son point de vue sur le monde. C'est l'acte d'écriture qui compte donc le plus. Comme le remarque Julie Houle, l'espace géographique est placé en arrière-plan dans les récits de voyage de la fin du XXe siècle jusqu'au au début du XXI et laisse une plus grande place à la vision que les auteures élaborent de leur voyage :

Plusieurs voyageuses font preuve d'une certaine originalité en ayant recours à un discours beaucoup plus imagé, voire coloré, et à un langage empreint d'humour. Leur véritable innovation réside, en revanche, dans les rapports que les aventurières entretiennent avec l'espace où elles se déplacent. D'objet central du récit, l'espace devient, dans les écrits publiés entre 1980 et 2003, un décor dans lequel se déroule l'aventure. L'accent est véritablement mis sur la narration du périple. (p. 46)

Martel met donc en scène des sons et des scènes non pas tant pour décrire des espaces ou des lieux que pour souligner sa 
présence en tant que sujet, sa capacité à dire le monde et la réalité qui l'entoure. On voit ainsi que la relation de Martel à l'espace n'a pas la même valeur que pour Darveau. En effet, le voyage géographique dans Randonnée au pays des totems devient un voyage personnel dans Whisky berbère; en d'autres mots, un voyage vers soi-même, une quête de soi, d'où l'omniprésence de subjectivités et la mise en arrière-plan de l'espace géographique. L'espace et le déplacement dans l'espace sont pour elle un moyen de se rapprocher d'autrui afin de refaire, ré-imaginer sa subjectivité; c'est un moyen de transformer son identité. Selon Houle, les médias (télévision, cinéma, Internet), les avancées technologiques et le tourisme de masse participent tous à la démocratisation du voyage. Le déplacement n'est donc plus nécessaire pour "accéder à une certaine connaissance » de l'ailleurs (Houle, p. 6). On se déplace alors, comme avec Martel, afin d'en apprendre plus sur soi, d'être plus vrai, plus authentique si l'on peut dire, à partir de l'Autre.

\section{Structure et agencement du texte : un écho ostensible de la narration}

On constate sur le plan de la structure, en lisant le livre de Darveau, qu'elle prête à son récit une forme épistolaire par la disposition par entrées de journal et l'insertion de repères spatiotemporels comme titres (par exemple, WINNIPEG ET LES PRAIRIES, 3 juillet). Des photographies de paysages et de villes qu'elle a visitées agrémentent le texte au fur et à mesure des entrées qui leur correspondent. Cet agencement, qui fait appel à un deuxième médium, renforce l'impression d'être plus près d'une réalité dite objective, une recherche qui semble chère à 
l'auteure et qui, on l'a vu, s'exprime à travers sa narration. Il renforce aussi l'effet de réel bâti dans la narration en donnant aux lecteurs à voir autrement, et de manière peut-être plus tangible, ce voyage qu'elle a entrepris. Le titre, Randonnée au pays des totems, est aussi révélateur: il dénote un récit en premier lieu basé sur le déplacement géographique vers une altérité dont on occulte encore sa forme humaine afin de se concentrer sur ses aspects les plus impressionnants et les plus accessibles, les totems. On note aussi que l'auteure a édité ellemême son récit, mais au lieu de percevoir cela comme une aliénation de sa part, on peut le voir ici comme une stratégie qui lui permet de rester dans les normes tout en les ployant puisqu'une autoédition lui permet de transmettre ses expériences et son discours sans le filtre d'une institution littéraire fortement patriarcale. Ainsi, Darveau participe de cette manière à fonder une littérature des femmes. Le titre du récit, les photographies, les entrées, les repères spatiotemporels et l'autoédition renforcent l'autorité discursive de Darveau, car ils consolident l'effet de réel construit dans la narration.

Pour Martel, la forme qu'elle prête à son récit vient de la fiction et détient des caractéristiques à la fois du roman et du recueil de nouvelles. Par exemple, son récit est organisé par chapitres et ceux-ci portent des titres comme "Les cinq sens", "Le visage camouflé » ou encore «La chenille verte». Ceux-ci nous informent plus sur une histoire que sur un lieu en particulier qui fut visité, comme c'est le cas chez Darveau. La mise en scène du voyage paraît ainsi d'autant plus renforcée. À la suite de ces titres, on trouve en exergue des proverbes, des dictons marocains ou encore certaines morales qui lui furent partagées par les gens qu'elle a côtoyés lors de ses deux séjours 
au Maroc. Ce trait répond à la recherche et à la présence de l'altérité dans le récit et je note que l'aspect didactique de ces pensées mises en exergue semble plus s'adresser à l'auteure qu'aux lectrices et lecteurs, puisqu'on sait maintenant que le voyage correspond pour Martel à une quête de soi. On trouve aussi avant le texte une note qui informe que le "récit a été élaboré à partir de notes accumulées au cours de deux voyages au Maroc, le premier [...] [en] 2002 et le second en [...] 2010 » (p. 8; je souligne). Ces deux voyages, faits à huit années d'intervalle, en paraissent pourtant comme un seul dans la narration. Martel fournit ainsi une forme romanesque à son récit de voyage, car elle ne cherche pas à faire une transcription fidèle de ses périples, ce que dénote l'utilisation du terme « élaboré » dans la citation; elle veut au contraire conter son voyage, le refaire ou plutôt en faire une histoire. Cette mise en fiction indique que l'auteure se fie maintenant plus à ses propres références, à son propre système de valeurs et à son propre imaginaire, qu'elle s'approprie des éléments fictionnels afin de bâtir un récit de voyage. D'ailleurs le titre, Whisky berbère, au premier abord étonnant et énigmatique, devient porteur de sens lorsqu'on apprend dans le texte qu'il s'agit de la manière dont on nomme, au Maroc, le thé marocain. Il indique à quel point Martel fait une place en elle à l'altérité et à sa culture. Un lexique, une carte des lieux visités et une table de matières parachèvent le récit de l'auteure et contribuent à faire percevoir que l'élément le plus important, dans son voyage, est le voyage intérieur et non le voyage physique, géographique. En effet, c'est son histoire qui est mise à l'avant-plan tandis que les détails pratiques paraissent à la toute fin de son récit.

Le récit de voyage des femmes du Québec, à travers l'analyse que j'ai présentée, nous informe donc d'une autre 
manière d'explorer l'ailleurs et d'entrer en contact avec l'Autre. Chez Jacqueline Darveau et Marie-Ève Martel, il dénote une volonté de légitimer leur autorité discursive, ce qui consiste pour moi à visibiliser un esprit féminin en littérature et, pour Monicat, à " résister et à construire une autre histoire ». On a vu que cette subversion s'opère à partir de la narration et de la structure du texte qui se déploie différemment chez chaque auteure. À travers ces points de résistance, on a aussi remarqué que Darveau et Martel créent des lieux du savoir : la première valorise les écrivaines, les écrivains et les scientifiques, la précision et la sphère publique; la seconde indique que l'expérience humaine individuelle et les rencontres, amicales ou intimes sont des références valables. Ces lieux du savoir nous informent à leur tour de la conception du voyage et du récit de voyage chez les deux auteures. Pour Darveau, le voyage constitue une occasion de se tailler une place dans la société et l'écriture du voyage est une façon de visibiliser sa présence et de faire entendre sa voix publiquement. Pour Martel, le voyage représente une découverte de soi à travers l'Autre et l'écriture du voyage est une valorisation de son imaginaire, de sa capacité à légitimer sa perspective sur le monde. Une autre analyse qui opérerait à partir d'un corpus plus large pourrait confirmer si la narration et la forme structurelle sont des lieux de résistance spécifiques aux écrits de voyage écrit par les femmes du Québec. Elle pourrait aussi découvrir d'autres manières par lesquelles les femmes valorisent leur autorité discursive à travers ce genre littéraire. On pourrait aussi pousser plus loin l'analyse et en même temps la réflexion de Lanser en examinant si les récits de voyage des femmes (du Québec ou en général) se dotent d'une voix narrative qui leur sont propre étant donné que ce genre littéraire se situe entre le monde réel et fictionnel. 


\section{Bibliographie}

CARLE, Anne-Marie. (1999), «Écrire hors de la maison du père. Les récits des voyageuses canadiennes-françaises, 18591940 », mémoire de maîtrise, Sherbrooke, Université de Sherbrooke.

DARVEAU, Jacqueline. (1938), Randonnée au pays des totems, Québec, [Cie de l'Éclaireur].

HoUle, Julie. (2003), « Récits de voyageuses québécoises, 19802003 », mémoire de maîtrise, Sherbrooke, Université de Sherbrooke.

LANSER, Susan Sniader. (1992), Fictions of Authority: Women Writers and Narrative Voice, Ithaca, Cornell University Press.

MALENFANT, Brigitte. (1997), «L'horizon autobiographique du récit de voyage de Flora Tristan, Les pérégrinations d'une paria », mémoire de maîtrise, Sherbrooke, Université de Sherbrooke.

MARTEL, Marie-Ève. (2011), Whisky berbère, Montréal, Fides.

MoNICAT, Benedicte. (1996), Itinéraires de l'écriture au féminin : voyageuses du $19^{e}$ siècle, Amsterdam, Rodopi. 


\section{Résumé}

Le récit de voyage de Jacqueline Darveau, Randonnée au pays des totems (1938), et celui de Marie-Ève Martel, Whisky berbère (2011), témoignent de pratiques d'écriture très différentes. En effet, elles ne s'inscrivent pas dans le langage de la même façon afin de légitimer leur regard sur le monde. Nous avancerons l'hypothèse que ces voyageuses s'expriment différemment parce qu'elles ne construisent pas dans leur écriture les mêmes lieux du savoir. Nous nous attarderons au discours et à la forme pour comprendre comment les femmes du Québec consolident leur autorité discursive dans leur récit.

\section{Abstract}

The travel diary of Jacqueline Darveau, Randonnée au pays des totems (1938), and the one of Marie-Ève Martel, Whisky berbère (2011), testify of very different writing practices. Indeed, those authors do not invest language in the same way in order to legitimize their view of the world. We advance the hypothesis that these travelers express themselves differently because they do not build the same places of knowledge. We propose to focus on the discourse and the form to understand how women of Québec consolidate their discursive authority by writing their travel diary. 